\title{
Electrochemical quartz crystal microbalance study of the behaviour of bis(2,2-bipyridyl)copper(II) complex immobilized on a self-assembled monolayer modified gold electrode
}

\author{
Abdunnaser Mohamed Etorki, Haddi Omar Reghei \\ Department of Chemistry, University of Tripoli, Tripoli, Libya \\ Email address: \\ a.etorki@ch.uot.edu.ly (A. M. Etorki) \\ To cite this article: \\ Abdunnaser Mohamed Etorki, Haddi Omar Reghei. Electrochemical Quartz Crystal Microbalance Study of the Behaviour of Bis(2,2- \\ Bipyridyl)Copper(II) Complex Immobilized on a Self-Assembled Monolayer Modified Gold Electrode. American Journal of Physical \\ Chemistry. Vol. 3, No. 5, 2014, pp. 67-71. doi: 10.11648/j.ajpc.20140305.13
}

\begin{abstract}
Modification of a gold electrode of an electrochemical quartz crystal microbalance (EQCM) has been achieved by immobilizing a bis(2,2-bipyridyl)copper(II) complex on a self-assembled monolayer (SAM) of 1-mercapto-11-undecanoic acid (MUA). The electrostatic interaction of the negatively charged SAM with a di-positive copper complex allowed the attachment. The modified electrode exhibited excellent redox behavior. Electro-statically immobilized on a SAM gold modified electrode is reported. A carboxylic acid terminated monolayer was utilized to provide a chemically uniform surface. The dependence of the modified electrode response was investigated in terms of $\mathrm{pH}$, supporting electrolyte and ionic strength. X-ray photoelectron spectroscopy (XPS) was also employed to study the chemical interactions between bis(2,2bipyridyl)copper(II) and 1-mercapto-11-undecanoic acid (MUA). XPS provides identification of the sorption sites involved in the accumulation of bis(2,2-bipyridyl)copper(II), as well as the forms of species sorbed on the self-assembled monolayer. It is found that sorption occurs on carboxlic functional groups.
\end{abstract}

Keywords: Self-Assembled Monolayer, Quartz Crystal Microbalance, XPS, Adsorption, Modified Electrode

\section{Introduction}

Chemically modified electrodes have received growing interest in the last three decades. The main goal of this kind of electrode is to improve the selectivity and sensitivity of the electrochemical reactions that occur on their surfaces for different purposes, especially in analytical chemistry. Many different strategies have been employed for the electrode surface modification, such as adsorption, covalent bonding, polymer covering [1]. Self-assembled monolayer (SAM) systems offer a simple and effective method for modifying metal surfaces with desired properties. Molecular selfassembly has become a popular procedure for surface derivatization, mostly due to its simplicity, versatility, reproducibility and establishment of a high level of order on a molecular dimension [2]. These properties provide a necessary platform for probing the relationship between molecular microstructure on an electrode surface and macroscopic electrochemical properties such as ion barrier, electron tunneling, electrocatalysis and molecular sensing [3] In this modification process, a molecular layer spontaneously forms on a substrate which is immersed in a solution containing the adsorbate molecules. The reaction is driven by strong interaction between the substrate surface and a specific functional group of the adsorbate. Variation of the structure of the free terminal of the adsorbate allows the study of the solution/substrate interface. Functionalized SAMs have been used to bind cells and proteins to surfaces, producing optical diffraction gratings, to immobilize redox active probes to electrode surfaces in order to study electrontransfer reactions [4].

A variety of self-assembling systems have already been reported including chlorosilanes, fatty acids and alkanethiols. During the last decade, extensive research works have been directed to the preparation and characterization of welldefined monolayers on gold electrode surfaces using sulfur containing species, such as thiol and disulfides [5].

Moreover, alkanethiols terminated with functional groups, 
such as a carboxylic acid and an amine, are the most important for many applications [5]. Due to the specific functions of the terminal groups, they have been used to control surface properties and immobilize different molecules on the monolayer [6]. Mercaptopropionicacid has been known to act as a so-called promoter molecule, and a monolayer of MPA provided the functionality to facilitate the electron transfer reaction between cytochrome $c$ and the electrode [6]. Adsorption properties have been also studied for immobilization of proteins and other compounds onto MPA monolayers. Furthermore, an MPA monolayer has been used as an anchor layer to fabricate sensing electrodes, which can improve the stability and selectivity of composite sensing films such as enzyme/poly-L-lysine systems and enzyme-entrapped polyion complex-bilayer membranes [6]. It has been reported that an MPA monolayer is useful for maintaining the intrinsic activity of various enzymes and biological molecules [7].

In this paper, the electrochemical study of a bis $\left(2,2^{-}\right.$ bipyridyl) copper(II) chloride $\left\{\left[\mathrm{Cu}(\text { bipy })_{2}\right] \mathrm{Cl}_{2} \cdot 6 \mathrm{H}_{2} \mathrm{O}\right\}$ electrostatically immobilized on a SAM gold modified electrode is reported. A carboxylic acid terminated monolayer was utilized to provide a chemically uniform surface for further attachment. Copper (II) compounds have presented catalytic activity for important species with good possibilities for application in sensor development for biological molecules.

\section{Experimental}

\subsection{Reagents}

1-mercato-11-undecanoic acid (MUA), tris(hydroxymethyl)aminomethane-maleate (TRISMALEATE), tris(hydroxymethyl)aminomethane(TRIS), (hydroxethyl)piperazine-N ${ }^{-}$-2-ethanesulfonic acid (HEPES), BARBITAL, phosphate buffer, absolute ethanol, L-ascorbic acid, 4-acetamidophenol, citric acid, sodium salicylate, dimethylformanide (DMF), sulfuric acid, potassium chloride and $30 \% \mathrm{H}_{2} \mathrm{O}_{2}$ were chemicals of analytical grade from Aldrich -Sigma. $\left[\mathrm{Cu}(\text { bipy })_{2}\right] \mathrm{Cl}_{2} \cdot 6 \mathrm{H}_{2} \mathrm{O}$ was purchased from Fluka.

\subsection{Apparatus and Procedure}

All electrochemical measurements were carried out using a Reference 3000 Potentiostat (Gamry, USA). The working electrodes were gold quartz crystals of the electrochemical quartz crystal microbalance (EQCM, Gamry USA). The counter electrode was Pt gauze and the potentials were quoted vs. a saturated calomel electrode (SCE ) reference electrode. Cyclic voltammetry (CV) was performed from 0.2 to $0.8 \mathrm{~V}$ vs. SCE using the SAM modified electrodes in supporting electrolyte solution which contained $5 \mathrm{mM}$ $\mathrm{K}_{3} \mathrm{Fe}(\mathrm{CN})_{6} / 1 \mathrm{M} \mathrm{KCl}$ to investigate the quality and integrity of the SAM on the gold surface. The electrochemical readings were taken at the scan rates of $5,10,50,10 \mathrm{mV} \mathrm{s}^{-1}$. All experiments were performed under ambient conditions.
Solutions were purged with argon for 20 minutes before measurements and blanketed with argon during the measurements to prevent $\mathrm{O}_{2}$ redissolution.. Polished $10 \mathrm{MHz}$ AT-cut quartz crystals were used as supplied from International Crystal Manufacturing (ICM), and have a smaller than $1 \mu \mathrm{M}$ alumina abrasive finish. The piezoelectrically and electrochemically active areas are 0.21 $\mathrm{cm}^{2}$ and $0.23 \mathrm{~cm}^{2}$ respectively.

\subsection{Electrode Modification}

The electrode was abundantly rinsed with deionized water, and sonicated in water for $2 \mathrm{~min}$; finally the electrode were cleaned in freshly prepared 'piranha'solution (1:3, 30\% $\mathrm{H}_{2} \mathrm{O}_{2}$-conc. $\mathrm{H}_{2} \mathrm{SO}_{4}$ ) for $10 \mathrm{~min}$ and then washed by Milli-Q water (Millipore) and ethanol. Immediately after the cleaning step, the electrodes were transferred to a $10 \mathrm{mM}$ solution of MUA in ethanol (thiol solution).

To prepare the monolayer, the gold electrode was immersed into the thiol solution for $2 \mathrm{~h}$. The modified electrode was further rinsed with ethanol and water to remove physically adsorbed molecules, and then transferred to a solution of $\left.5 \mathrm{mg} \mathrm{ml}^{-1}[\mathrm{Cu} \text { (bipy })_{2}\right]$ in $\mathrm{DMF}$, for $15 \mathrm{~min}$. The bare gold electrode and the electrode just modified by MUA were used for the experimental control.

\section{Results and Discussion}

Fig. 1 shows the cyclic voltammograms (CVs) of the bisbipy copper(II) complex adsorbed on a bare (a) and an MUA modified (b) electrode, recorded at $50 \mathrm{mV} \mathrm{s}^{-1}$ in $0.01 \mathrm{~mol}$ $\mathrm{dm}^{-3}$ HEPES buffer, $\mathrm{pH}$ 7.0. Successful incorporation of the redox complex in the SAM modified electrode surface is evidenced by the presence of well defined anodic $(300 \mathrm{mV})$ and cathodic $(200 \mathrm{mV})$ peaks [8].

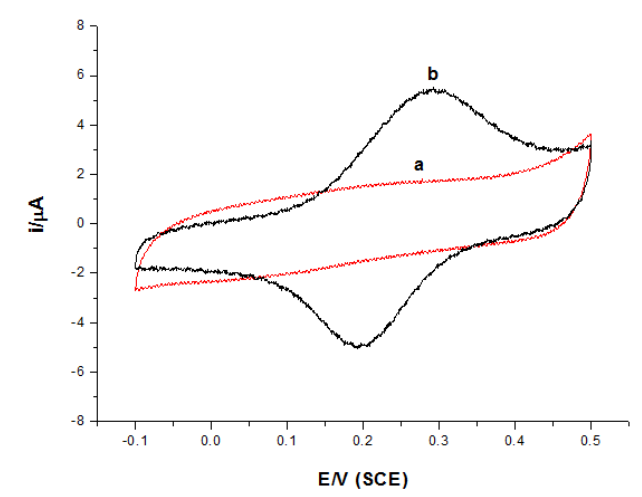

Figure 1. CVS of the [ Cu(bipy) 2$]$ / SAM modified gold electrode (b) and (a) bare gold electrode in $0.01 \mathrm{M}$ buffer $(\mathrm{pH}=7.0)$, scan rate $50 \mathrm{mV \textrm {s } ^ { - 1 }}$.

The plot of anodic $\left(\mathrm{Ip}_{\mathrm{a}}\right)$ and cathodic peak $\left(\mathrm{Ip}_{\mathrm{c}}\right)$ presents in Figure 2 and yielded a straight line, this along with the surface coverage estimated by integrating the area under the voltammetric peak (corresponding to a charge of $19.7 \mu \mathrm{C}$ $\mathrm{cm}^{-2}$ ) being practically the same for both the anodic and cathodic peaks, confirms that the voltammetric behavior of the modified electrode is due to a reversible surface redox 
reaction [9].

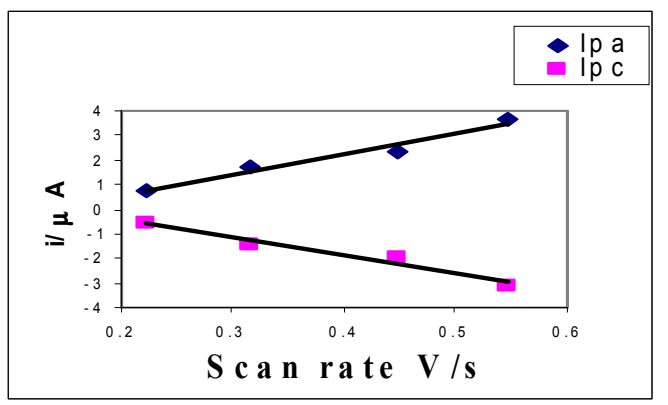

Figure 2. Plot of the anodic (Ipa) and cathodic (Ipc) peak currents against potential Scan rate. Conditions were similar to that in Figure 1.

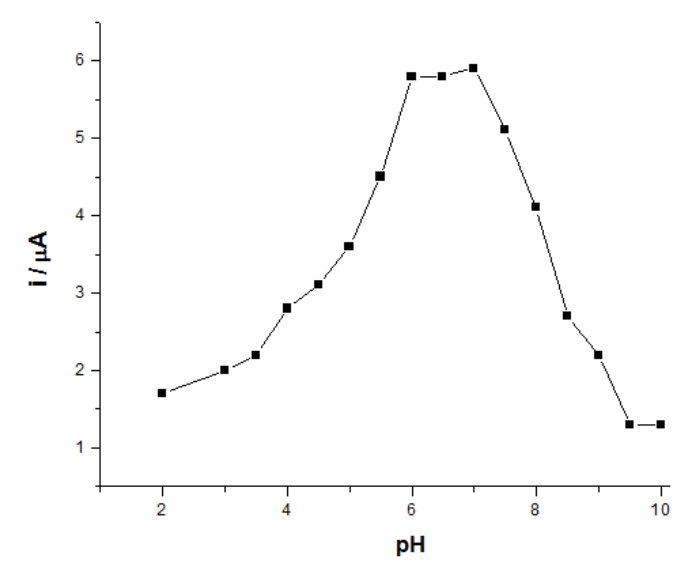

Figure 3. The effect of $\mathrm{pH}$ on the modified electrode. Conditions were Similar to that in Figure 1.

For an ideal, reversible redox behavior of the adsorbed species, $\Delta E \mathrm{p}$ should be zero and independent of the scan rate. However, most of the experiments reported, nearly reversible redox systems are, indeed, characterized by finite values of $\Delta E \mathrm{p}$. The studies on $\Delta E \mathrm{p}$ variation as a function of scan rate on this modified electrode indicate that $\Delta E \mathrm{p}$ increases to a little extent (e.g., from 50 to $75 \mathrm{mV}$, using HEPES buffer) as scan rate is increased from 50 to $200 \mathrm{mV} \mathrm{s}^{-1}$. The low values of $\Delta E \mathrm{p}$, which change slightly with the scan rate, suggest that the modification yields an almost reversible surface-bound redox system. The half-peak width $\left(\Delta E_{1 / 2}\right)$ of the voltammetric waves is predicted to be $90.6 / n \mathrm{mV}$ under ideal conditions where the adsorbates do not interact with each other. In the present case, values of 95 and $90 \mathrm{mV}$ for the anodic and cathodic peaks have been observed, respectively, using HEPES buffer as supporting electrolyte. The electron transfer rate constant for the immobilized complex, evaluated by the Laviron method, had a value of $1.94 \mathrm{~s}^{-1}$, indicating a good reversibility and showing a fast electron transfer of the electrochemical reaction in the diffusionless electrochemical system. The surface property of a SAM is affected by its charged state, as is known, the carboxylic acid terminal group in MUA exhibits $\mathrm{pH}$ dependence. At low $\mathrm{pH}$ range the SAM surface should be fully protonated without net charge. In contrast, the terminal group of the SAM molecules dissociates in solutions with high $\mathrm{pH}$, which charges negatively. Since the bis-bipy copper(II) complex is attached to the SAM electrode surface by electrostatic interactions, $\mathrm{pH}$ control is of great importance. The effect of the solution $\mathrm{pH}$ on the modified electrode is shown in Fig. 3.

The electrode shows the best signal at $\mathrm{pH}$ 7. The drawback for $\mathrm{pH}>7$ is due to $\mathrm{OH}^{-}$, which leads to a ternary hydroxocomplex formation, as the $\mathrm{OH}^{-}$concentration in solution increases, the fraction of simple $\left.[\mathrm{Cu} \text { (bipy })_{2}\right]$ diminishes.

The type of the supporting electrolyte has an important effect on the performance of the $\left[\mathrm{Cu}(\text { bipy })_{2}\right] / \mathrm{SAM}$ modified electrode, thus five different supporting electrolyte were tested. Fig. 4 shows the response of the modified electrode in HEPES, phosphate, $\mathrm{KCl}$, TRIS-MALEATE and TRIS buffers

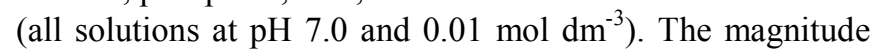
order of the response is as follows: HEPES $\geq \mathrm{KCl}>$ TRISMALEATE $>$ phosphate $>$ PIPES $>$ TRIS.

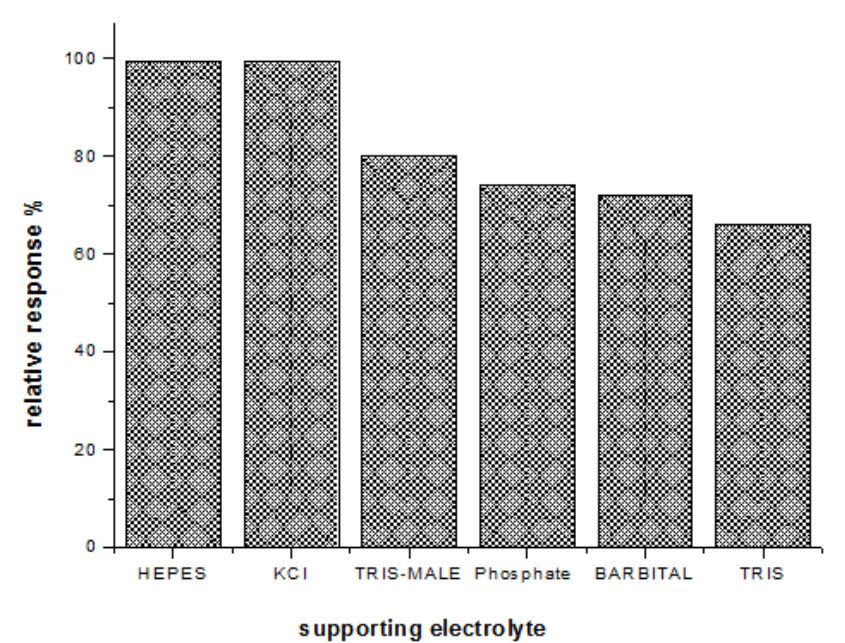

Figure 4. Relative response of the [Cu(bipy) $\left.{ }_{2}\right] / S A M$ modified gold electrode in different supporting electrolytes. Conditions were similar to that in Figure 1.

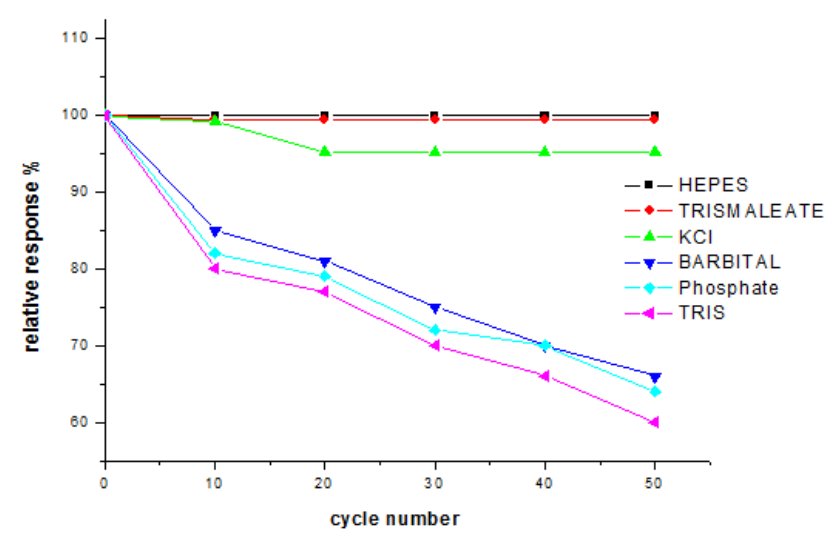

Figure 5. Stability of the $[\mathrm{Cu}($ bipy) 2$] / \mathrm{SAM}$ modified gold electrode in different Supporting electrolytes as a function of the number of cycles.

Fig. 5 shows continuous cycling of the modified electrode in all buffer solutions in the potential range from $100 \mathrm{mV}$ up to $400 \mathrm{mV}$ for about $180 \mathrm{~min}$. An important factor in choosing the supporting electrolyte is the compromise between its buffering capability and its interaction with the complex. For example, it is well known that phosphate has a 
high affinity for copper ions; this kind of interaction could lead to a replacement in the coordinated ligands. HEPES buffer was chosen as the standard supporting electrolyte, since it promoted good electrochemical characteristics and stability, which are important for analytical proposes.

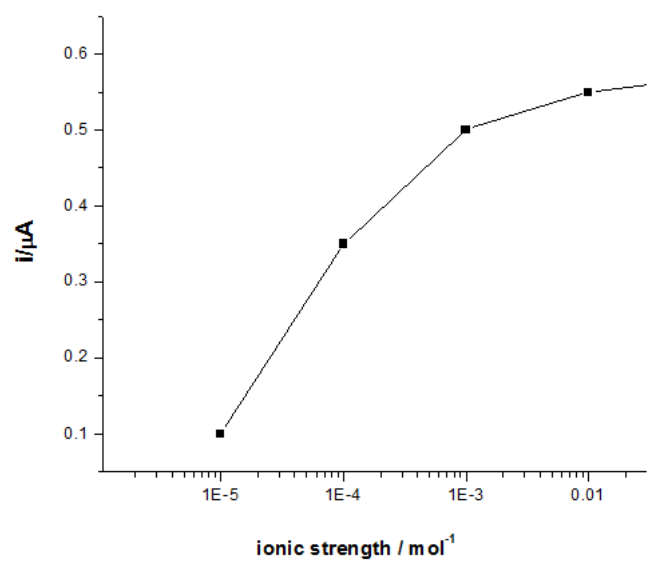

Figure 6. Dependence of the [Cu(bipy) $\left.{ }_{2}\right] / S A M$ modified gold electrode on the ionic strength. Conditions were similar to that Figure 1.

Fig. 6 presents the influence of ionic strength of the HEPES buffer solution at $\mathrm{pH} 7.0$ on the response of the modified electrode. Once more, since the copper complex is electrostatically attached to the SAM electrode surface, a high ionic strength could interfere in the immobilization stability. As it can be observed, the electrostatically driven adsorption of $\left[\mathrm{Cu}(\text { bipy })_{2}\right]$ on the MUA/SAM is not limited in high ionic strength solutions, but the current decreased with ionic strength values lower than $3.5 \times 10^{-3} \mathrm{~mol} \mathrm{dm}^{-3}$, probably due to the increase in the resistance of the electrochemical cell. Further experiments were all carried out at $3.5 \times 10^{-3} \mathrm{~mol} \mathrm{dm}^{-3}$, which was chosen as the optimum ionic strength to achieve the highest current intensity.
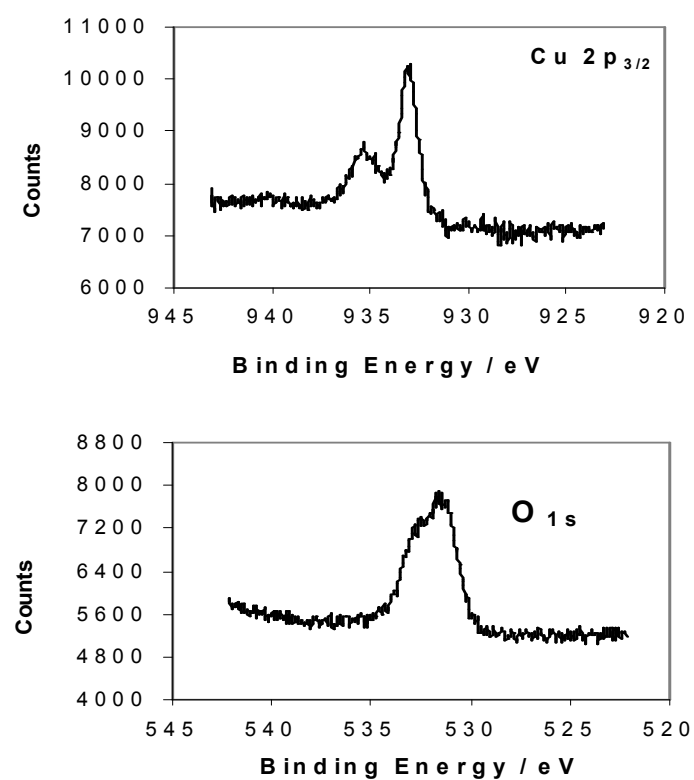

Figure 7. XPS spectra of the [Cu(bipy) 2$] / S A M$ modified gold electrode. Conditions were similar to that in Figure 1.
The nature of the chemical interaction between the $\mathrm{Cu}$ (II) and carboxylate groups of the MUA SAM can be seen in the Figure 7. $\mathrm{Cu}\left(2 \mathrm{p}_{3 / 2}\right)$ XPS region in the first peak at $933.0 \mathrm{eV}$ is characterized as the alginate-bound $\mathrm{Cu}$ (II) and the second peak at $934.8 \mathrm{Cu}(\mathrm{II})$ originated from $\mathrm{Cu}(\mathrm{II})$ located in the coordination form (copper carboxylate). The results of peak fitting for $\mathrm{O}_{(1 \mathrm{~s})}$ illustrate two distinct chemically shifted $\mathrm{O} 1 \mathrm{~s}$ core level emissions occurring at 533.3 and $532.0 \mathrm{eV}$ with a relative intensity ratio of $1: 1$ assigned to hydroxyl and carbonyl oxygen atoms, respectively. The $1.3 \mathrm{eV}$ binding energy separations is within the range of chemical shift reported for carboxylic acid functions.

\section{Conclusions}

The electrostatic interaction of the $\operatorname{bis}\left(2,2^{-}-\right.$ bipyridyl)copper(II) complex with a self-assembled monolayer of 1-mercato-11-undecanoic acid (MUA) offers a simple and effective method for modifying a gold electrode with the desired electrocatalytic properties. The modified electrode exhibited an excellent redox behavior and good electrocatalytic activity. The experiments described above illustrate the ability to employ this modified electrode in future for biomolecules detection with excellent sensitivity, selectivity and precision.

\section{References}

[1] A. D’Annibale, R. Regoli, P. Sangiorgio and T. Ferri, Electroanalysis, 1999, 11, 505.

[2] D. Mandler and I. Turyan, Electroanalysis, 1996, 8, 207.

[3] C. R. Raj and T. Ohsaka, J. Electroanal. Chem., 2001, 496, 44.

[4] T. Sawaguchi, Y. Sato and F. Mizutani, J. Electroanal. Chem., 2001, 507, 256.

[5] P.D.Ding, L.M.Niu, H.Q.Luo and N.B.Li, Croat.Chem.Acta, $2007,80,61$.

[6] K. Bandyopadhyay and K. Vijayamohanan, Langmuir, 1998, 14,625 .

[7] C. N. Sayre and D. M. Collard, Langmuir, 1997, 13, 714.

[8] T. Sawaguchi, Y. Sato and F. Mizutani, J. Electroanal. Chem., 2001,507, 256.

[9] A. Ulman, An Introduction to Ultrathin Organic Films From Langmuir Blodgett to Self-Assembly, Academic Press, San Diego, CA, 1991.

[10] S. A. John, F. Kitamura, K. Tokuda and T. Ohsaka, J. Electroanal Chem., 2000, 492, 137.

[11] M. J. Tarlov and E. F. Bowden, J. Am. Chem. Soc., 1991, 113, 1847.

[12] M. Collinson, E. F. Bowden and M. J. Tarlov, Langmuir, 1992, $8,1247$.

[13] S. Arnold, Z. Q. Feng, T. Kakiuchi, W. Knoll and K. Niki, J. Electroanal. Chem., 1997, 438, 91. 
71 Abdunnaser Mohamed Etorki and Haddi Omar Reghei: Electrochemical Quartz Crystal Microbalance Study of the Behaviour of Bis(2,2-Bipyridyl)Copper(II) Complex Immobilized on a Self-Assembled Monolayer Modified Gold Electrode

[14] B. L. Frey, C. E. Jordan, S. Kornguth and R. M. Corn, Anal. Chem., 1995, 67, 4452.

[15] F. Mizutani, Y. Sato, Y. Hirata, T. Sawaguchi and S. Yabuki, Anal. Chim. Acta, 1998, 364, 173.

[16] F. Mizutani, S. Yabuki, Y. Sato, T. Sawaguchi and S. Iijima, Electrochim. Acta, 2000, 45, 2945.

[17] D. N. Upadhyay, V. Yegnaraman and P. G. Rao, Langmuir, 1996, 12, 4249.

[18] L.Zhu, K.Zhao, K.Wang, H.Xian, Z.Shang and W.Sun, Sensors, 2008,8,5649.

[19] J. W. Zhao, L. Q. Luo, X. R. Yang, E. K. Wang and S. J. Dong, Electroanalysis, 1999, 11, 1108.

[20] S. Kortly and L. Sucha, Handbook of Chemical Equilibrium in Analytical . Chemistry, Ellis Horwood, New York, 1985
[21] I. Fabian, Inorg. Chem., 1989, 28, 3805.

[22] B Tolbert, American Chemical Society, Washington,DC, 1982101-123

[23] P. J. O'Connell, C. Gormally, M. Pravda and G. G. Guilbault, Anal. Chim. Acta, 2001, 431, 239.

[24] J. C. B. Fernandes, L. T. Kubota and G. Oliveira-Neto, Electroanalysis, 1999, 11, 475.

[25] M. H. Pournaghi-Azar, H. Razmi-Nerbin and B. Hafezi, Electroanalysis, 2002, 14, 206.

[26] P. J. O'Connell, C. Gormally, M. Pravda and G. G. Guilbault, Anal. Chim. Acta, 2001, 431, 239. 